\title{
HIGH VOLTAGE LIMITS FOR ELECTROSTATIC TRANSDUCERS: ON THE RoLE OF AMBIENT PRESSURE AND DEVICE DIMENSION IN MICRODISCHARGES
}

\author{
Chester G. Wilson ${ }^{1}$, Amy E. Wendt ${ }^{1}$, and Yogesh B. Gianchandani ${ }^{1,2}$ \\ ${ }^{1}$ Department of Electrical and Computer Engineering, University of Wisconsin, Madison, WI 53706 \\ ${ }^{2}$ EECS Department, University of Michigan, Ann Arbor, MI 48109
}

\begin{abstract}
In order to understand the details of high-field breakdown in microstructures that are vacuum packaged, a series of experiments are used to determine characteristics of microdischarges. It is found that many of these contradict conventional assumptions based upon large scale discharges. When planar microelectrodes are used, Paschen's curve is not followed; the anode and cathode current paths are a strong function of pressure; voltage gradients are supported in the glow region; and the glow region is confined directly over the cathode. It is shown that magnets can be used to further confine the discharge. This information is pertinent for recently reported devices that exploit microdischarges for sensing.
\end{abstract}

\section{INTRODUCTION}

Electrostatic sensing and actuation are pervasive in MEMS technology not only because of the wide variety of materials that they accommodate, but also because they are generally power efficient [1-4]. In many cases performance measures such as sensitivity and dynamic range of sensors, or force and displacement of actuators are limited by the highest electric field that can be safely achieved, or voltage bias that can be applied. While there has been an incipient effort to study this phenomena at atmospheric pressure [5], most such devices are packaged in vacuum. In addition, the spatial distribution of the electric field and breakdown current, which are critical parameters for sustained avalanche breakdown, have not been reported in the context of microstructures. By addressing these questions, this paper attempts to establish design guidelines for high field devices. In doing so it also addresses the requirements for sustained arcs and microplasmas which have been successfully used etch silicon wafers and sense chemical impurities in liquids and gases [6,7]. The experiments demonstrate that microdischarges violate many of the assumptions used routinely for larger scale discharges.

\section{STRUCTURE AND EXPERIMENT}

It is conventionally believed that the breakdown voltage (BV) in a vacuum environment follows the Paschen curve (Fig. 1) which indicates that a minimum voltage is achieved at a particular product of pressure and separation between bias electrodes $(P \bullet d)$. This is constrained by fewer collisions at lower pressure and lower collisional energy at higher pressures, both of which necessitate a higher electric field to sustain the avalanche breakdown. However, with most microstructures, multiple path lengths are simultaneously available, which makes the discharge gap a variable, and permits a low BV to be sustained even as the pressure changes from the value that favors the minimum electrode separation (Fig. 1).

In order to measure the spatial distribution of discharge current, a discharge was created between planar thin-film metal electrodes on a glass substrate at various levels of vacuum. Segmented electrodes (Fig. 2) were first used as a cathode, and then as an anode to measure the difference between the bias-dependent trajectory of the positive ions and electrons respectively, in a nitrogen ambient. Figure 3 shows that the cathode current favors longer paths at 1.2 Torr, but at 6 Torr the distribution between the four pads is comparable. In contrast, the same change in pressure results in a more dramatic change in the spatial distribution of anodic current, (Fig 3). This asymmetry is believed to be caused by secondary emission electrons, which are abundant near the cathode, in sustaining microdischarges, as discussed next.

Another important aspect of microdischarges that differs from conventional assumptions is that the glow region is confined to the proximity of the cathodes (Fig 5). This suggests that the ionization events in microplasmas are driven primarily by secondary electrons emitted by the cathodes. In contrast, larger scale plasmas have a glow region that extends between the electrodes, with a prominent dark sheath near the cathode. This phenomenon can be critical to the design of microdischarge-based sensors and display devices that have recently been reported [7-10]. In addition, the voltage in the glow region of large-scale plasmas is nearly constant. Most of the voltage drop is across the sheath near the cathode, which has a diminished electron population. Measurements of the potential distribution of microdischarges were performed using the arrangement shown in Fig 6. In contrast to large scale plasmas, microdischarges show a significant voltage gradient (electric field) in the glow region over the cathode, which ranges from $0.10-0.25 \mathrm{~V} / \mu \mathrm{m}$ over 1.2-6 Torr (Fig. 7,8).

By using concentric cylindrical magnets located beneath a planar electrode, we have been able to tailor the shape of the glow region, demonstrating that the microdischarge mechanism can be controlled in a spatially selective manner (Fig 9).

In conclusion, these results demonstrate how breakdown in vacuum varies with design parameters and operating conditions, and that it can be substantially different from conventional assumptions.

\section{ACKNOWLEDGEMENTS}

This effort is supported in part by the National Science Foundation, US Geological Survey, and the Sea Grant. Partial travel support for the conference was provided by the Transducers Research Foundation, and by the DARPA MEMS and DARPA BioFlips programs.

\section{REFERENCES}

[1] L.J.Hornbeck, "Current status of the digital micromirror device (DMD) for projection television applications, IEEE IEDM, 1993, pp. 381-4

[2] B.Gogoi, C.Chuan, C.Mastrangelo, "Force balanced micro-machined pressure sensors," IEEE T-ED, 48(8), 8/01, pp. 1575-84

[3] T.Tsuchiya, Y.Kageyama, H.Funabashi, J.Sakata, "Polysilicon vibrating gyroscope vacuum encapsulated in an on-chip microchamber," Sensors \& Actuators, A90(1-2), 5/01, pp. 49-55

[4] F.Ayazi, H.H.Chen, F.Kocer, H.Guong, K.Najafi, "A HARPSS polysilicon vibrating ring gyroscope," JMEMS, 6/01, pp. 169-79

[5] T.Ono, Y.Dong, M.Esashi, "Microdischarge and electric breakdown in a micro-gap," $J M \& M, 10(3) 9 / 00$, pp. 445-51

[6] C.Wilson, Y.Gianchandani, "Silicon micro-machining using in-situ DC microplasmas," JMEMS 10(1) 3/01, p. 50-4

[7] J.C.T. Eijkel, H. Stoeri, A. Manz, "A dc microplasma on a chip employed as an optical emission detector for gas chromatography," Anal. Chem. 72, 6/00, pp. 2547-2552

[8] C.Wilson, Y.Gianchandani, "LEd-SpEC: Spectroscopic detection of contaminants using glow discharges from liquid microelectrodes," IEEE MEMS Conf., Las Vegas, 1/02, pp. 248-51

[9] G.Jenkins, A.Manz, "Optical emission detection of liquid analytes using a micro-machined D.C. glow discharge device at atmospheric pressure" -TAS, 2001, pp. 349-350

[10] S.J.Park, J.Chen, C.Liu, J.G.Eden, "Silicon microdischarge devices having inverted pyramidal cathodes: Fabrication and performance of arrays," Appl. Phys. Lett., (78)4, 1/01, pp. 419-21 


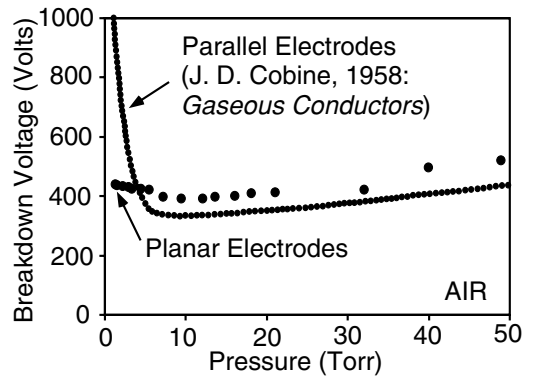

Fig. 1:

Paschen

voltage

breakdown

curve for

parallel and

planar

electrodes

with $500 \mu \mathrm{m}$

spacing.
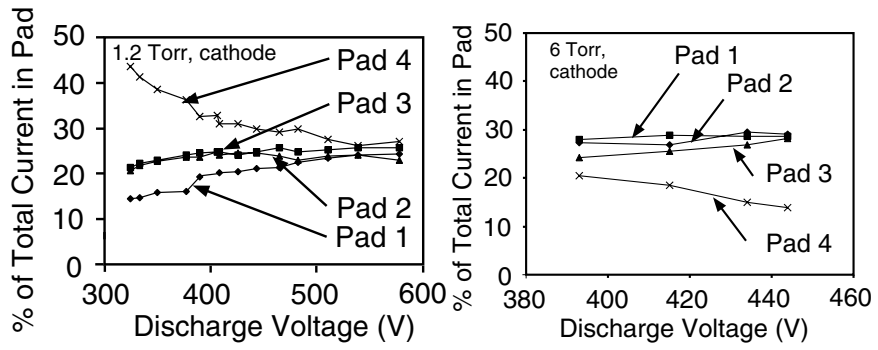

Fig. 3: Normalized current in the cathode electrode for an $N_{2}$ microplasma, operated at 1.2 Torr (a-left) and at 6 Torr (b-right).

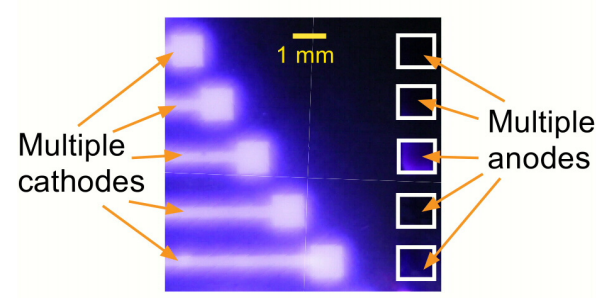

Fig. 5: Air microplasmas with varying electrode spacing show that the glow exists only over the cathode, and is independent of spacing, but dependent upon pressure and power density.

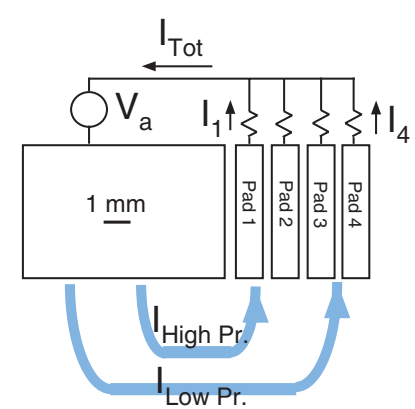

Fig. 2: Planar Ti electrode configuration, patterned on glass to measure cathode current density. Cathode is split into separate paths, where the current is independently measured. Polarity can be reversed to measure anode current density.
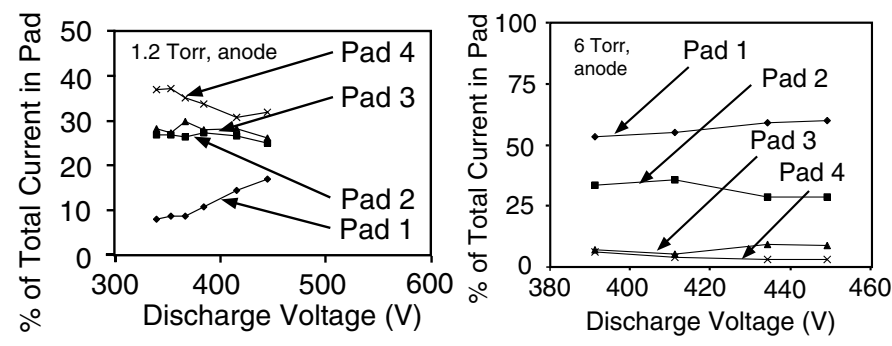

Fig. 4: Normalized current in the anode electrode for an $\mathrm{N}_{2}$ microplasma, operated at 1.2 Torr (a-left) and 6 Torr (b-right).

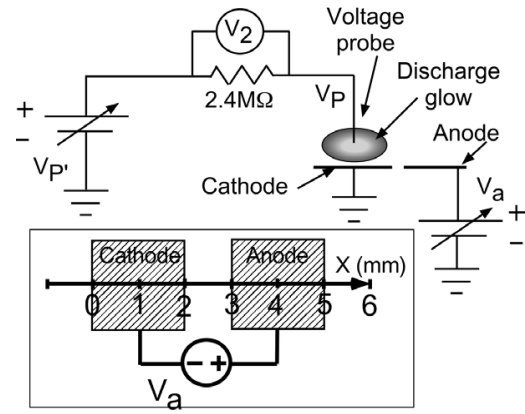

Fig. 6: Plasma floating potential $\mathrm{V}_{\mathrm{p}}$, is measured by varying applied voltage $\mathrm{V}_{\mathrm{P}}$, to find the value at which $\mathrm{V}_{2}$ is zero. The inset shows the dimensions of the planar electrodes.
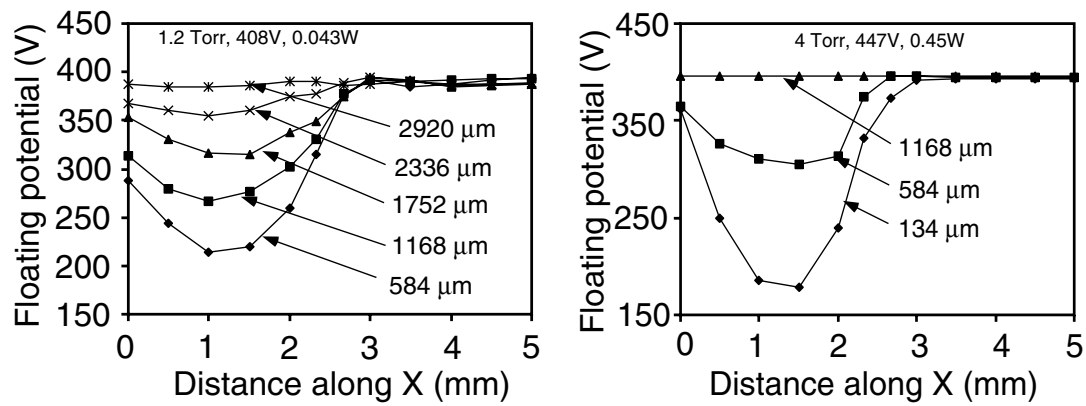

Fig. 7: Floating potential of an $\mathrm{N}_{2}$ microplasma at 1.2 Torr (a-left) and 4 Torr (b-right), generated by the electrodes shown in Fig. 6. Voltage is measured along $\mathrm{X}$ for varying heights over the coplanar electrodes. The plasma becomes more confined at higher pressures.

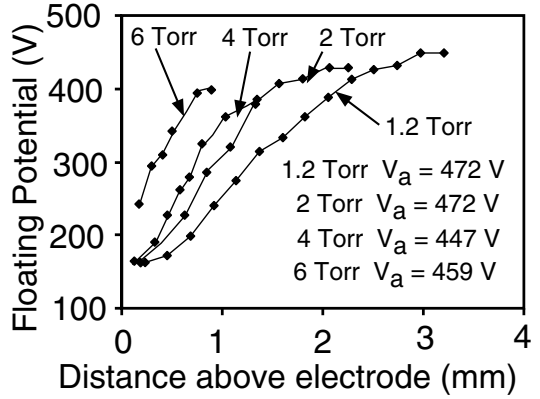

Fig. 8: Floating potential of $\mathrm{N}_{2}$ microplasmas at varying pressures, as a function of above electrodes at $\mathrm{X}=1.2$ $\mathrm{mm}$. Glow height decreases and voltage gradient increases w/ increasing pressure.

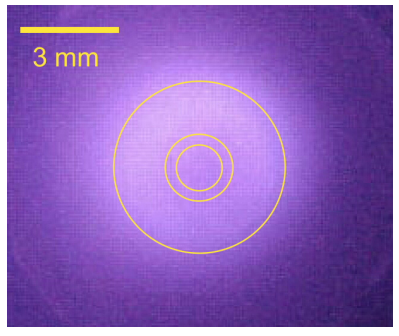

Fig. 9: Microplasma at 1.2 Torr confined using a coaxial magnetic structure. 